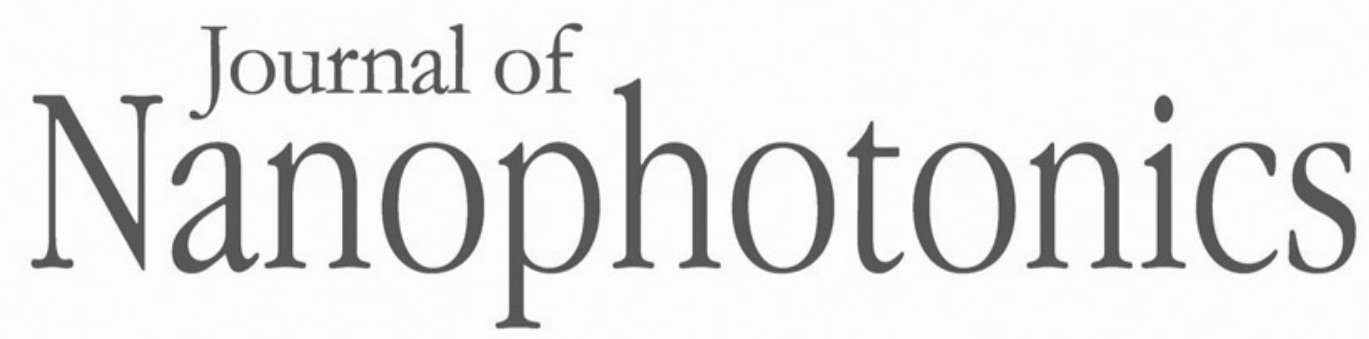

Nanophotonics.SPIEDigitalLibrary.org

\title{
Special Section Guest Editorial: Advances in Nanostructured Thin Films
}

\author{
Akhlesh Lakhtakia \\ Tom G. Mackay \\ Motofumi Suzuki
}




\title{
Special Section Guest Editorial: Advances in Nanostructured Thin Films
}

\author{
Akhlesh Lakhtakia, ${ }^{a}, *$ Tom G. Mackay, ${ }^{b}, *$ and Motofumi Suzuki ${ }^{c}, *$ \\ ${ }^{a}$ The Pennsylvania State University, Department of Engineering Science and Mechanics, \\ University Park, Pennsylvania 16802, United States \\ ${ }^{b}$ University of Edinburgh, School of Mathematics and Maxwell Institute for \\ Mathematical Science, James Clerk Maxwell Building, King's Buildings, \\ Edinburgh, EH9 3FD, United Kingdom \\ ${ }^{\mathrm{c}}$ Kyoto University, Department of Micro Engineering, Kyoto daigaku-Katsura, \\ Nishikyo-ku, Kyoto 615-8540, Japan
}

With ingenuity and fortitude, the structures of thin films can be engineered at the nanometer length scale. The control over the optical and photonic properties of thin films that is thereby afforded may be usefully exploited for a host of practical applications, including optical sensing, photovoltaic electricity generation, the creation of biomimetic components, optical coatings, and so forth, as well as harnessed for fundamental investigations into the nature of light-matter interactions. Societal benefits may follow in the shape of devices with enhanced performances, lower costs, and lesser environmental impacts, not to mention the general cultural benefits that flow from the advancement of science and engineering.

It is within this context that the eighth annual Conference on Nanostructured Thin Films took place in San Diego on the 12th and 13th of August 2015 as part of the annual SPIE Optics + Photonics symposium. For the past eight years, this conference has provided a forum for the nanostructured-thin-films community to present and discuss its latest research findings. As in previous conferences, the 2015 conference featured a diverse mix of presentations pertaining to the design, fabrication, and characterization of nanostructured thin films, delivered by both well-established and up-and-coming researchers, representing all parts of the world. In total, 39 presentations were delivered, spread over 10 oral sessions and one poster session, on topics such as control and modification, applications and devices, theoretical studies, and fabrication. The conference began with a keynote lecture on optical coatings and metamaterials.

The following special section of the Journal of Nanophotonics comprises papers either based on presentations at Nanostructured Thin Films VIII or closely related to presentations at the conference. These papers provide a bird's-eye view of current advances relating to the optics and photonics of nanostructured thin films, and illustrate the vitality of this rapidly developing field of research.

\section{Acknowledgments}

The guest editors thank their program committee, all authors for their contributions to this special section, the reviewers, and all those who participated in the Nanostructured Thin Films VIII conference and contributed to its proceedings.

Akhlesh Lakhtakia is the Charles Godfrey Binder (Endowed) Professor of Engineering Science and Mechanics at The Pennsylvania State University. His current research interests include electromagnetic surface waves, circular polarization and Beltrami fields, thin-film solar cells, sculptured thin films, mimumes, bone refacing, bioreplication, and forensic science. He became a Fellow of SPIE in 1996.

\footnotetext{
*Address all correspondence to: Akhlesh Lakhtakia, E-mail: akhlesh@psu.edu; Tom G. Mackay, E-mail: T.Mackay@ed.ac.uk; Motofumi Suzuki, E-mail: m-snki@me.kyoto-u.ac.jp

(๑) 2016 Society of Photo-Optical Instrumentation Engineers (SPIE)
} 
Tom G. Mackay is a reader in the School of Mathematics at the University of Edinburgh, and also an adjunct professor in the Department of Engineering Science and Mechanics at The Pennsylvania State University. His current research interests include homogenization, complex materials, and sculptured thin films. He became a Fellow of SPIE in 2010.

Motofumi Suzuki is a professor at Kyoto University. He received his bachelor's, master's, and doctor of engineering degrees from Kyoto University in 1986, 1988, and 1998, respectively. His current research interests include the optical properties of nanostructured thin films and the synthesis of novel nanostructures. He is a member of SPIE. 